\title{
Algebraic structures on the topology of moduli spaces of curves and maps
}

\author{
Y.-P. Lee and R. Vakil
}

\begin{abstract}
We discuss selected topics on the topology of moduli spaces of curves and maps, emphasizing their relation with Gromov-Witten theory and integrable systems.
\end{abstract}

\section{Contents}

0. Introduction 197

1. Integrable systems 200

2. Faber-type Gorenstein conjectures, and three sisters 201

3. Polynomiality 203

4. Teleman's work on family topological field theory 207

5. Witten's conjecture on $\boldsymbol{r}$-spin curves 213

$\begin{array}{ll}\text { References } & 216\end{array}$

\section{Introduction}

Forty years ago, Deligne and Mumford introduced their celebrated compactification of the moduli space of curves. In 1983, Mumford [Mu] initiated a comprehensive study of intersection theory on the moduli space of curves, and in particular introduced the tautological ring. In many ways motivated by Witten's conjecture [W1], the last twenty years have seen a steadily growing understanding of rich algebraic structures on the cohomology of moduli spaces of curves, and related spaces, such as Gromov-Witten invariants and Hurwitz numbers. Even when the structures deal purely with the moduli space of curves, Gromov-Witten theory has provided a powerful tool for understanding them. The purpose of this article is to discuss some of these structures.

1. Integrable systems. Witten's conjecture determines all top intersections of $\psi$-classes on $\overline{\mathcal{M}}_{g, n}$, by showing that their generating function satisfies

(C)2009 International Press 
differential equations coming from integrable systems (the KdV hierarchy, or the Virasoro algebra). More precisely, let

$$
F_{g}=\sum_{n \geq 0} \frac{1}{n !} \sum_{k_{1}, \ldots, k_{n}}\left(\int_{\overline{\mathcal{M}}_{g, n}} \psi_{1}^{k_{1}} \cdots \psi_{n}^{k_{n}}\right) t_{k_{1}} \cdots t_{k_{n}},
$$

and let $F=\sum F_{g} \hbar^{2 g-2}$ be the generating function for all genera. Then the $\mathrm{KdV}$ form of Witten's conjecture is:

$$
\begin{aligned}
& (2 n+1) \frac{\partial^{3}}{\partial t_{n} \partial t_{0}^{2}} F \\
& \quad=\left(\frac{\partial^{2}}{\partial t_{n-1} \partial t_{0}} F\right)\left(\frac{\partial^{3}}{\partial t_{0}^{3}} F\right)+2\left(\frac{\partial^{3}}{\partial t_{n-1} \partial t_{0}^{2}} F\right)\left(\frac{\partial^{2}}{\partial t_{0}^{2}} F\right)+\frac{1}{4} \frac{\partial^{5}}{\partial t_{n-1} \partial t_{0}^{4}} F .
\end{aligned}
$$

Define differential operators $(n \geq-1)$

$$
\begin{aligned}
L_{-1}= & -\frac{\partial}{\partial t_{0}}+\frac{\hbar^{-2}}{2} t_{0}^{2}+\sum_{i=0}^{\infty} t_{i+1} \frac{\partial}{\partial t_{i}} \\
L_{0}= & -\frac{3}{2} \frac{\partial}{\partial t_{1}}+\sum_{i=0}^{\infty} \frac{2 i+1}{2} t_{i} \frac{\partial}{\partial t_{i}}+\frac{1}{16} \\
L_{n}= & \sum_{k=0}^{\infty} \frac{\Gamma\left(m+n+\frac{3}{2}\right)}{\Gamma\left(k+\frac{1}{2}\right)}\left(t_{k}-\delta_{k, 1}\right) \frac{\partial}{\partial t_{n+k}} \\
& +\frac{\hbar^{2}}{2} \sum_{k=1}^{n-1}(-1)^{k+1} \frac{\Gamma\left(n-k+\frac{1}{2}\right)}{\Gamma\left(-k-\frac{1}{2}\right)} \frac{\partial}{\partial t_{k}} \frac{\partial}{\partial t_{n-k-1}} \quad(n>0)
\end{aligned}
$$

These operators satisfy $\left[L_{m}, L_{n}\right]=(m-n) L_{m+n}$. The Virasoro form of Witten's conjecture, due to Dijkgraaf, Verlinde, and Verlinde, is: $L_{n} e^{F}=0$ for all $n$. These relations inductively determine the coefficients of $F$, and hence compute all intersection numbers.

This unexpected relationship with integrable systems has since appeared repeatedly, and we now have a modest understanding as to why this relationship might exist. This subject is well-covered in the literature, so we content ourselves with a short discussion in $\S 1$.

2. Faber-type Gorenstein conjectures. Based on numerical data found using Witten's conjecture, Faber made a remarkable conjecture $[\mathbf{F 1}]$ on the tautological part of the cohomology of $\mathcal{M}_{g}$. Again, this structure was quite unexpected, and in some sense we still have little understanding "why" such a structure should exist. This conjectural structure seems to be shared by related moduli spaces. This topic is also well-covered (see for example Pandharipande's ICM talk $[\mathbf{P a}])$, so we describe just enough in $\S 2$ to motivate later discussion.

3. Polynomiality. Proof of parts of the above conjectures often come through another "polynomiality" structure of certain invariants or intersection numbers, which is exemplified in their first appearance, the ELSV 
formula [ELSV1, ELSV2]. For example, the polynomiality of the ELSV formula is an easy-to-state fact about factoring permutations into transpositions in the symmetric group, but the only known proof requires the geometry of the moduli space of curves. There are other instances that look similar that are even less understood. In $\S 3$, we discuss the notion of polynomiality, and describe and explain a conjecture of Goulden, Jackson, and the second author that is sketched in [GJV1]: that there should be an ELSV-type formula relating certain double Hurwitz numbers to the intersection theory of some moduli space. We then describe work of Shadrin and Zvonkine $[\mathbf{S Z}]$ and Shadrin $[\mathbf{S 1}]$ that these conjectural intersection numbers (arising from actual Hurwitz numbers) have much richer structure than suspected in [GJV1].

4. Family topological field theory. Madsen and Weiss' celebrated proof [MW] of Mumford's conjecture on the stable (tautological) cohomology of the moduli space of curves has had an unexpected and powerful application to Gromov-Witten theory. Teleman has classified all cohomological 2D field theories based on a semi-simple complex Frobenius algebra $A$ : they are controlled by a linear combination of $\kappa$-classes and by an extension datum to the Deligne-Mumford boundary. This leads to a proof of Givental's conjecture that, roughly speaking, higher genus invariants are determined by genus 0 invariants. This in turn implies the Virasoro conjecture for manifolds with semisimple quantum cohomology. We outline this important work in some detail in $\S 4$.

5. Witten's conjecture on $r$-spin curves. In the last section, we briefly outline Faber-Shadrin-Zvonkine's proof [FSZ] of Witten's conjecture on $r$-spin curves, emphasizing on the parts which are most related to the tautological rings. Although this result is a consequence of Teleman's theorem, it uses completely different ingredients and the proof itself is quite interesting in its own right.

Two ingredients in their proof will be briefly explained:

(1) The first author's theorem that tautological relations hold for generating functions of axiomatic (semisimple) theories [L2].

(2) Faber-Shadrin-Zvonkine's reconstruction theorem which states that the Witten's correlators in higher genus can be uniquely reconstructed from genus zero.

Along the way, we also give some explanation of Givental's formalism ([Gi2, $\mathbf{L P}]$ and references therein) which has proved important in understanding the algebraic structure of Gromov-Witten theory. Note that the proof of the first result can be reduced to an elementary statement in the geometry of boundary divisors on moduli of curves via Givental's theory. See Section 5.3.

Notation and background. We work over $\mathbb{C}$. General background may be found in $[\mathbf{M S}$, Ch. $23-25],[\mathbf{V}]$, and $[\mathbf{L 4}]$. We assume the reader is familiar with the following notions: 
(i) the moduli space of pointed curves $\mathcal{M}_{g, n}$ and its partial compactifications $\mathcal{M}_{g, n}^{r t}$ (curves with rational tails), $\mathcal{M}_{g, n}^{c t}$ (curves of compact type), and $\overline{\mathcal{M}}_{g, n}$ (Deligne-Mumford stable curves, the topic of this volume).

(ii) the cohomology classes $\psi_{i}(1 \leq i \leq n), \kappa_{j}$, and $\lambda_{k}(0 \leq k \leq g)$, and the tautological ring.

We also note that [S2] contains an excellent review of basic notions of cohomological field theories and Givental theory.

Acknowledgements. We would like to thank T. Jarvis, K. Liu, S. Shadrin, C. Teleman, and D. Zvonkine for their helpful comments on earlier versions of the paper.

Both authors are partially supported by the NSF.

\section{Integrable systems}

We content ourselves with a brief overview of Witten's conjecture and related topics, sufficient to set the stage for later sections.

It was only since the advent of Witten's conjecture that we are able to compute all top intersections of $\psi$-classes in $\overline{\mathcal{M}}_{g, n}$, something we now take for granted. These allow one to compute all top intersections in the tautological ring, $[\mathbf{F 2}]$. It is also important when considering Witten's conjecture to know not just that it is true, but also why it is true, i.e., why in retrospect integrable systems should be expected to control these top intersections. Of course, Witten's original heuristic argument is one explanation.

There are now a large number of proofs of Witten's conjecture, and it is a sign of the richness of the conjecture that almost every proof has been fundamentally new, with fundamentally new insights. Kontsevich's original proof $([\mathbf{K o}]$, see also $[\mathbf{L o 1}])$ remains unlike the others. Mirzakhani's proof [Mi] (the third) gives an elegant interpretation of each summand in the Virasoro version of Witten's conjecture.

The remaining proofs pass through the ELSV formula (discussed in $\S 3$ ), counting branched covers, and fundamentally about factoring permutations in the symmetric group. Okounkov and Pandharipande's proof $[\mathbf{O P}]$ was part of their massive program in Gromov-Witten theory, including their proof of the Virasoro conjecture in dimension 1.

By a careful algebraic argument, Kim and K. Liu $[\mathbf{K i L i}]$ give a direct proof of Witten's conjecture through localization. Chen, Li, and Liu thereafter gave a different and very short proof, using ideas from [GJV2] on the $\lambda_{g}$-conjecture (a sister conjecture to Witten's conjecture, see the next section).

Meanwhile, Kazarian and Lando also gave an algebro-geometric proof of Witten's conjecture [KaLa]. Shortly after that, also using ideas from [GJV2], Kazarian gave a greatly streamlined proof $[\mathbf{K a}]$. 
At each stage, our understanding of the structure behind Witten's conjectured deepened, and in some sense we now have quite a strong understanding of why it is true.

One test of our understanding is how well it generalizes to other situations. We briefly mention two generalizations of Witten's conjecture that will be relevant shortly.

First, Witten gave a generalization of his conjecture to the moduli space of $r$-spin curves [W2]. Roughly speaking, an $r$-spin curve with marked points is an $r$ th root of the canonical bundle twisted by given multiples of the points; see $\S 5.2$ for more detail.

The Virasoro conjecture (mentioned earlier), due to Eguchi, Hori, Xiong, and also S. Katz gives Virasoro-related constraints on the Gromov-Witten invariants of a complex projective manifold [CK, §10.1.4]. The case of a point is the Virasoro form of Witten's conjecture.

More relatives of Witten's conjecture will appear in the next section.

\section{Faber-type Gorenstein conjectures, and three sisters}

Faber's conjectures are well exposed in the literature; for example, the second author has discussed them in detail in $[\mathbf{V}, \S 3.2]$. So again we content ourselves with saying just enough to continue our story.

2.1. Faber's conjectures. Faber's conjectures [F1] describe an unexpected and in many ways still-unexplained structure on the tautological ring $R^{*}\left(\mathcal{M}_{g}\right)$, which may be considered as a subring of the cohomology ring or the Chow ring (although this technically yields two different conjectures).

Informally speaking, Faber's conjectures on $\mathcal{M}_{g}$ state that $R^{*}\left(\mathcal{M}_{g}\right)$ behaves like the cohomology ring of a $(g-2)$-dimensional complex projective manifold. Somewhat more precisely, this means:

I. "Vanishing/socle" conjecture. $R^{i}\left(\mathcal{M}_{g}\right)=0$ for $i>g-2$, and $R^{g-2}\left(\mathcal{M}_{g}\right)$ $\cong \mathbb{Q}$. This was proved by Looijenga $[$ Lo2 $]$ and Faber $[$ F1, Thm. 2].

II. Perfect pairing conjecture. The analogue of Poincaré duality holds: for $0 \leq i \leq g-2$, the natural map $R^{i}\left(\mathcal{M}_{g}\right) \times R^{g-2-i}\left(\mathcal{M}_{g}\right) \rightarrow R^{g-2}\left(\mathcal{M}_{g}\right) \cong \mathbb{Q}$ is a perfect pairing. This is currently open.

III. Intersection number conjecture. Faber gives a formula for top (i.e., total degree $g-2$ ) intersections of generators of the tautological ring, as a multiple of a generator of $R^{g-2}\left(\mathcal{M}_{g}\right)$. As we discuss in $\S 2.3$, this is proved.

The three conjectures above completely determine the tautological ring $R^{*}\left(\mathcal{M}_{g}\right)$.

There are three "sister" conjectures that parallel Faber's, on three different spaces. See $[\mathbf{P a}]$ for more on the three sisters, and a more detailed history.

2.2. The first sister: $\overline{\mathcal{M}}_{\boldsymbol{g}, \boldsymbol{n}}$. On $\overline{\mathcal{M}}_{\boldsymbol{g}, n}$, there is an analogous set of conjectures, with $g-2$ replaced by $3 g-3+n$. This was first asked as 
a question by Hain and Looijenga [HLo, Question 5.5]; first stated as a speculation by Faber and Pandharipande [FP1, Speculation 3] (in the case $n=0$ ), and first stated as a conjecture by Pandharipande [Pa, Conj. 1]. In the cohomology ring, I and II trivially hold, but in the Chow ring, this is far from clear. (I was first shown in [GV1].) Witten's conjecture should be considered the intersection number part III.

2.3. The second sister: $\mathcal{M}_{\boldsymbol{g}}$. The pointed version of Faber's conjecture $\mathcal{M}_{g}$ isn't for $\mathcal{M}_{g, n}$; it should be for $\mathcal{M}_{g, n}^{r t}$, the space of curves with "rational tails" (those stable curves with a component of geometric genus $g$ ). In this case, the role of $g-2$ is replaced by $g-2+n$. The intersection number portion of the conjecture (often called Faber's intersection number conjecture) may be stated as follows: If all $a_{i}>0$, then

$$
\pi_{*} \psi_{1}^{a_{1}} \cdots \psi_{n}^{a_{n}}=\frac{(2 g-3+n) !(2 g-1) ! !}{(2 g-1) ! \prod_{j=1}^{n}\left(2 a_{j}-1\right) ! !} \kappa_{g-2} \quad \text { for } \sum a_{i}=g-2+n .
$$

(This implies part III of Faber's conjecture, see above, hence we use the same name.) This is now a theorem. Getzler and Pandharipande showed that the statement is a formal consequence of the Virasoro conjecture for $\mathbb{P}^{2}[\mathbf{G e P}]$, in fact the large volume limit. Givental thereafter described a strategy for a proof of the Virasoro conjecture for projective space, and more generally Fano toric manifolds [Gi1, Gi2]. A complete write-up was promised in $[\mathbf{L P}]$ a number of years ago, but it has not yet appeared. Subsequently, a different proof and a complete solution to Givental's conjecture was given by C. Teleman $[\mathbf{T}]$, which in particular proves the Virasoro conjecture in the semisimple case. The proof of Teleman depends crucially on Mumford's conjecture, proved by Madsen and Weiss. This is a powerful approach to Faber's conjecture, but perhaps somewhat roundabout. Recently, K. Liu and Xu have given an stunningly short and direct proof $[\mathbf{L X}]$. Their approach is quite different, and is part of their larger program for understanding the algebraic structure of these intersection numbers. Most notably, their approach yields a surprisingly simple explicit formula for Witten's $n$-point function, and this has produced a number of interesting new results about intersection numbers, of which Faber's intersection number conjecture is just one.

As with Witten's conjecture, the susceptibility of this problem to different approaches illustrates the richness of the question. One should hope that the story is not yet over, and more results should be obtainable from the successful earlier approaches. Furthermore, the beautiful form of equ. (2.1) clearly suggests that there should be a strong reason for it, and that further understanding should be sought. (The connection to double Hurwitz numbers, explored in [GJV3], seems to be deeply related to this question.)

2.4. The third sister: the $\lambda_{\boldsymbol{g}}$-conjecture, for $\mathcal{M}_{\boldsymbol{g}, \boldsymbol{n}}^{\text {ct }}$. The third sister of Faber's conjecture is for the space $\mathcal{M}_{g, n}^{c t}$, the space of curves of 
compact type (stable curves whose dual graphs have no loop; those stable curves with compact Jacobian). The corresponding conjecture is due to Faber and Pandharipande ([FP1, Spec. 2], [Pa, Conj. 1]), with $g-2$ is replaced by $2 g-3$. The socle portion was first proved as a consequence of Theorem * in [GrV2].

The intersection number portion of the conjecture was first conjecture by Getzler and Pandharipande $[\mathbf{G e P}]$. It is called the $\lambda_{g}$-conjecture because of its incarnation as a statement about intersections on $\overline{\mathcal{M}}_{g, n}$ (rather than $\left.\mathcal{M}_{g, n}^{c t}\right):$ For $n, g \geq 1$,

$$
\int_{\overline{\mathcal{M}}_{g, n}} \psi_{1}^{b_{1}} \cdots \psi_{n}^{b_{n}} \lambda_{g}=\left(\begin{array}{c}
2 g-3+n \\
b_{1}, \ldots, b_{n}
\end{array}\right) c_{g}
$$

where $\sum_{i=1}^{n} b_{i}=2 g-3+n, b_{1}, \ldots, b_{n} \geq 0$, and $c_{g}$ is a constant depending only on $g$.

The $\lambda_{g}$-conjecture has intriguingly proved more tractable than its sister intersection number conjectures, and a number of proofs now exist. When first proposing the conjecture, Getzler and Pandharipande showed that it is a formal consequence of the Virasoro conjecture for $\mathbb{P}^{1}$, which is now known (for example, by foundational work of Okounkov and Pandharipande). But before then, Faber and Pandharipande gave the first proof of the $\lambda_{g}$-conjecture, $[\mathbf{F P 2}]$. K. Liu, C.-C. M. Liu, and Zhou gave a new proof $[\mathbf{L L Z}]$ as a consequence of their proof of the Mariño-Vafa formula. Finally, Goulden, Jackson, and the second author gave a short direct (Gromov-Witten-free) proof in [GJV2] by exploiting the "polynomiality" structure described in the next section, using the ELSV-formula (also described shortly).

Kazarian and, independently at the same time, Kim and K. Liu showed that the algebraic structure introduced in [GJV3], properly understood, also yield proofs (and explanations) of Witten's conjecture. (This insight was certainly not known to the authors of [GJV3].) Their two proofs (the most recent and shortest proofs, mentioned above) are quite distinct, and insightful. A complete understanding of the algebraic structures underlying Witten's conjecture would presumably involve putting these two proofs into a common larger framework.

\section{Polynomiality}

We next describe the phenomenon of polynomiality of quasi-enumerative problems on moduli spaces of curves and maps. The central motivating example is the ELSV formula.

3.1. The ELSV formula. Fix a genus $g$, a degree $d$, and a partition of $d$ into $n$ parts, $\alpha_{1}+\cdots+\alpha_{n}=d$, and let $r=2 g+d+n-2$. Fix $r+1$ distinct points $p_{1}, \ldots, p_{r}, \infty$ on $\mathbb{P}^{1}$. Define the Hurwitz number $H_{\alpha}^{g}$ as the number of branched covers of $\mathbb{P}^{1}$ by a (connected) Riemann surface, that are unbranched away from $p_{1}, \ldots, p_{r}, \infty$, such that the branching 
over $\infty$ is given by $\alpha_{1}, \ldots, \alpha_{n}$ (the monodromy lies in the conjugacy class corresponding to that partition), and the branching over each $p_{i}$ is $2+1+$ $\cdots+1=d$ (the simplest nontrivial branching). We consider the $n$ preimages of $\infty$ to be labeled.

Up to a straightforward combinatorial factor, this corresponds to the answer to the following combinatorial problem: given a permutation in conjugacy class $\alpha$, in how many ways can it be factored into $r$ transpositions that "connect" the numbers 1 through $n$ (transitive factorizations). (If the condition of transitivity seems unnatural, it is straightforward to connect to this to the problem without the transitive condition. This is equivalent to counting potentially disconnected covers. The algebraically simplest way to relate them: the exponential of the generating function counting connected covers is the generating function counting potentially disconnected covers.)

Based on extensive evidence, the combinatorialists Goulden and Jackson [GJ1, Conj. 1.2] had conjectured that this combinatorial problem had a surprising polynomial behavior: fixing $g$ and $n, H_{\alpha}^{g}$ is a simple combinatorial term times a symmetric polynomial in $\alpha_{1}, \ldots, \alpha_{n}$, with components in homogeneous degree between $2 g-3+n$ and $3 g-3+n$. This strongly suggests a connection between this combinatorial problem and the moduli space of curves!

Ekedahl, Lando, M. Shapiro, and Vainshtein explained this polynomiality with their ground-breaking ELSV-formula:

$$
H_{\alpha}^{g}=r ! \prod_{i=1}^{n}\left(\frac{\alpha_{i}^{\alpha_{i}}}{\alpha_{i} !}\right) \int_{\overline{\mathcal{M}}_{g, n}} \frac{1-\lambda_{1}+\cdots+(-1)^{g} \lambda_{g}}{\left(1-\alpha_{1} \psi_{1}\right) \cdots\left(1-\alpha_{n} \psi_{n}\right)}
$$

Here the denominator should be considered formally inverted, i.e., 1/(1$\left.\alpha_{i} \psi_{i}\right)=1+\alpha_{i} \psi_{i}+\alpha_{i}^{2} \psi_{i}^{2}+\cdots$, and the integral sign means to take the degree of the codimension $3 g-3+n$ (dimension 0) part of the integrand. Expanding the integral in the ELSV formula yields

$$
\sum_{a_{1}+\cdots+a_{n}+k=3 g-3+n}\left((-1)^{k} \int_{\overline{\mathcal{M}}_{g, n}} \psi_{1}^{a_{1}} \cdots \psi_{n}^{a_{n}} \lambda_{k}\right)\left(\alpha_{1}^{a_{1}} \cdots \alpha_{n}^{a_{n}}\right)
$$

and thus the polynomiality is explained by interpreting these numbers as top intersections on the moduli space of curves!

Better yet, the highest-degree terms are precisely the subject of Witten's conjecture, and indeed the five proofs of Witten's conjecture using the ELSV formula involve in different ways getting at these leading coefficients by asymptotic methods. Furthermore, the lowest-degree terms are precisely the subject of the $\lambda_{g}$-conjecture, which provides the entree for the proof of [GJV2].

This polynomiality arises repeatedly, usually as a result of localization on spaces of stable maps, and was a key ingredient in, for example, work of Graber and the second author, e.g., [GV1, GrV2]. 
By comparison, we give an example of polynomiality which has yet to be satisfactorily explained geometrically, which allows us to clarify a vague but suggestive conjecture of [GJV1].

3.2. A fourth sister? Conjectural geometry behind polynomiality of double Hurwitz numbers, [GJV1], and work of Shadrin and Zvonkine. We recall the history of the ELSV formula as motivation: we begin with an enumerative problem in geometry, of Hurwitz numbers, which can also be interpreted in terms of the combinatorics of the symmetric group. For fixed $g$ and $n$, these numbers are (up to a combinatorial factor) a polynomial in the parts of a partition (appearing in the definition of Hurwitz number). This polynomial is symmetric of degree $3 g-3+n$, which is highly suggestive of the moduli space of curves, and indeed there is a genus $g, n$ pointed curve present in the enumerative problem. The generating function for these numbers is constrained by the KP (Kadomtsev-Petviashvili) hierarchy; to see this, a change of variables is necessary. Thanks to the ELSV formula, these numbers are related to the intersection theory of a fundamental moduli space, and as a result, surprising structure is known on the (tautological) cohomology ring of the moduli space.

The identical story will apply in the situation we now describe, except that there is as of yet no moduli space, and no ELSV-type formula. There seems strong circumstantial evidence that there is a moduli space completing the story; this is the content of Conjecture 3.5 of [GJV1].

Instead of "single" Hurwitz numbers, we consider "one-part double" Hurwitz numbers $H_{(d), \beta}^{g}$, defined in the same way as single Hurwitz numbers, except that we require in addition complete branching over the point $0 \in \mathbb{P}^{1}$. Then by means of character theory, [GJV1, Thm. 3.1] shows that for fixed $g$ and $n, H_{(d), \beta}^{g}$ is a symmetric polynomial in $\beta_{1}, \ldots, \beta_{n}$, whose homogeneous pieces have even degree up to $4 g-3+n$.

The fundamental question this suggests is: is there a moduli space and ELSV-type formula explaining this polynomiality?

Note that there is a moduli space of dimension $4 g-3+n$ "present" in the problem: an $n$-pointed genus $g$ curve, along with a choice of line bundle. Motivated by this, one possible answer is the following.

Conjecture [GJV1, Conj. 3.5]. For each $g \geq 0, n \geq 1,(g, n) \neq$ $(0,1),(0,2)$,

$$
H_{(d), \beta}^{g}=r_{(d), \beta}^{g} ! d \int_{\overline{\operatorname{Pic}}_{g, n}} \frac{\Lambda_{0}-\Lambda_{2}+\cdots \pm \Lambda_{2 g}}{\left(1-\beta_{1} \psi_{1}\right) \cdots\left(1-\beta_{n} \psi_{n}\right)}
$$

where $\overline{\mathrm{Pic}}_{g, n}, \psi_{i}$, and $\Lambda_{2 k}$ satisfy a number of reasonable properties. For example, $\overline{\mathrm{Pic}}_{g, n}$ should be a compactification of the universal Picard variety over $\mathcal{M}_{g, n}$, which supports a (possibly virtual) fundamental class of the "expected" dimension $4 g-3+n . \psi_{i}$ should be an extension of the pullback of $\psi_{i}$ from $\mathcal{M}_{g, n}$. See [GJV1] for a complete list of proposed properties. 
It should be emphasized that the fundamental question is that of finding the right space, and this conjecture should be seen as merely a proposal. Some of the properties suggested by geometry yield testable constraints on double Hurwitz numbers (such as the string and dilaton equation), and these indeed hold [GJV1, Prop. 3.10].

Shadrin and Zvonkine show much more in $[\mathbf{S Z}]$ and $[\mathbf{S 1}]$. We emphasize that independent of the conjecture, their results are meaningful statements about double Hurwitz numbers. But their unpacking of the structure of these Hurwitz numbers is predicted precisely by the form of the conjecture. We state their results imprecisely in order to emphasize their form. Let $H$ be the generating function for the double Hurwitz numbers $H_{(d), \beta}^{g}$. Let $U$ be the generating function for just the highest-degree terms in the conjecture (i.e., those with no $\Lambda_{i}$ terms for $i>0$ ). Shadrin and Zvonkine define a linear differential operator $L$ which arises naturally in the cut-and-join formalism $\left(L=\sum b p_{b} \frac{\partial}{\partial p_{b}}\right.$, where $p_{b}$ tracks parts of $\alpha$ of size $\left.b\right)$.

The KP hierarchy is a system of partial differential equations on a power series $F$ in an infinite set of variables $p_{1}, p_{2}, \ldots$ The first three equations are:

$$
\begin{aligned}
F_{2,2}= & -\frac{1}{2} F_{1,1}^{2}+F_{3,1}-\frac{1}{12} F_{1,1,1,1} \\
F_{3,2}= & -F_{1,1} F_{2,1}+F_{4,1}-\frac{1}{6} F_{2,1,1,1} \\
F_{4,2}= & -\frac{1}{2} F_{2,1}^{2}-F_{1,1} F_{3,1}+F_{5,1}+\frac{1}{8} F_{1,1,1}^{2}+\frac{1}{12} F_{1,1} F_{1,1,1,1} \\
& -\frac{1}{4} F_{3,1,1,1}+\frac{1}{120} F_{1,1,1,1,1,1}
\end{aligned}
$$

Subscript $i$ refers to differentiation by $p_{i}$. The exponent $\tau=e^{F}$ of any solution is called a $\tau$-function of the hierarchy. For readable expositions on the KP hierarchy, see $[\mathbf{K a}, \S 6]$ and (influenced by this) [GJ2].

Shadrin and Zvonkine prove that under a scaling and renaming of variables, (i) $L^{2} H$ is a $\tau$-function of the KP hierarchy, i.e., it satisfies the bilinear Hirota equations. Furthermore, $L^{2} H$ satisfies the linearized KP equations. (ii) $U$ is a $\tau$-function for the KP hierarchy (in unusual variables), and thus satisfies the bilinear Hirota equations. Furthermore, it satisfies the linearized $\mathrm{KP}$ equations in the same variables. This is a complete analogue of Witten's Conjecture. Statement (i) follows in a standard way from the general theory of integrable systems, but the proof of (ii) is quite subtle.

Shadrin has recently taken this further. In [S1], he applies Kazarian's techniques to this series, relating the conjectured "intersection numbers" to the Hirota equations. The computations turn out to be simpler, and he gives explicit and rather transparent formulas for the generating series.

One might take Shadrin and Zvonkine's results to suggest that the moduli space behind the integrable system is precisely that suggested by [GJV1, Conj. 3.5]. However, this may not be the case: their methods are quite robust, 
and similar spaces (for example, with $n+1$ marked points, with the last point the preimage of 0 ) should yield similar results.

\section{Teleman's work on family topological field theory}

4.1. Terminology. For this section only, dimensions here mean dimensions over $\mathbb{R}$ and surfaces mean topological oriented 2-dim $\mathbb{R}$ "manifolds with nodal singularities". Curves, however, mean algebraic (or complex) curves!

4.2. Topological field theory and moduli of curves. C. Teleman introduced the notion of family topological field theory (FTFT) into GromovWitten theory. In the semisimple case, he completely classified all FTFTs. As an important application, he proved Givental's conjecture $[\mathbf{G i 2}]$ on the quantization formula, which in particular gives an explicit reconstruction of higher genus semisimple Gromov-Witten theory from the genus zero theory.

Teleman's result is the first instance of applying two powerful results from topology of moduli spaces of curves to Gromov-Witten theory: Harer stability and the Madsen-Weiss theorem (Mumford's conjecture). Harer stability is purely topological in its formulation, and Mumford's conjecture so far has no algebro-geometric proof. In this section, we will explain how these two results can be applied to Gromov-Witten theory.

4.3. Two dimensional topological field theory and semisimplicity. A 2- $\operatorname{dim}_{\mathbb{R}}$ topological field theory (TFT) is a symmetric, (strong, monoidal) functor of topological tensor categories $Z: \mathbf{C} \rightarrow$ Vec. Vec is the usual tensor category of vector spaces. $\mathbf{C}$ is the category whose objects are $1-\operatorname{dim}_{\mathbb{R}}$ oriented closed manifolds, i.e., a disjoint union of oriented $S^{1}$ 's. The morphisms are oriented cobordisms of the objects, with obvious compositions. The tensor structure is defined by disjoint union of objects.

The notion of TFT is equivalent to the notion of Frobenius algebra. A (commutative) Frobenius algebra is a $k$-algebra with an identity 1, a pairing $(\cdot, \cdot): A^{\otimes 2} \rightarrow A$ which is symmetric and nondegenerate, and satisfies the Frobenius property

$$
(a * b, c)=(a, b * c),
$$

where $*$ stands for the multiplicative structure of $A$.

The equivalence of TFT and Frobenius algebra can be seen as follows. $A=Z\left(S^{1}\right)$, and the identity element $1 \in A$ is defined by $Z$ (cap), (0 input, 1 output). The nondegenerate pairing $(\cdot, \cdot)$ is defined by $Z$ (bent cylinder) $(2$ inputs, 0 output), and the ring structure is defined by $Z$ (pair of pants) ( 2 inputs, 1 output). 


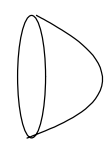

cap

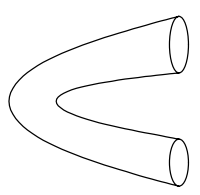

bent cylinder

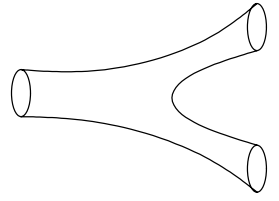

pair of pants

time direction

We will mainly be concerned with the semisimple case, where the product structure is diagonalizable. That is, $A \cong \oplus_{i} k \epsilon_{i}$ as an $k$-algebra, where $\epsilon_{i} * \epsilon_{j}=\delta_{i j} \epsilon_{i}$ is the canonical basis. Therefore, up to isomorphisms, $A$ is classified by $\Delta_{i}:=\left(\epsilon_{i}, \epsilon_{i}\right)$. Let

$$
\tilde{\epsilon}_{i}:=\frac{1}{\sqrt{\Delta_{i}}} \epsilon_{i}
$$

be the normalized canonical basis.

Proposition 4.1. Let ${ }^{q} C_{g}^{p}$ be an oriented surface of genus $g$, with $p$ inputs and $q$ outputs. Then $Z\left({ }^{q} C_{g}^{p}\right)$ defines a map

$$
{ }^{q} \mu_{g}^{p}: A^{\otimes p} \rightarrow A^{\otimes q},
$$

such that $\mu_{g}^{p}$ is "diagonal" in the (tensor power of the) normalized canonical basis. Furthermore, the entry of $\mu_{g}^{p}$ in $\tilde{\epsilon}_{i}^{\otimes p} \mapsto \tilde{\epsilon}_{i}^{\otimes q}$ is $\Delta_{i}^{\chi\left({ }^{q} C_{g}^{p}\right) / 2}$, where $\chi\left({ }^{q} C_{g}^{p}\right)$ is the (topological) Euler characteristic of ${ }^{q} C_{g}^{p}$.

This proposition can be easily proved by decomposing ${ }^{q} C_{g}^{p}$ into pairs of pants and applying the facts that $Z\left({ }^{1} C_{0}^{1}\right)$ is the identity and that $Z\left({ }^{1} C_{0}^{2}\right)$ defines the algebra multiplication and is diagonal with entries $\Delta_{i}^{1 / 2}$ in the normalized canonical basis.

Let ${ }^{1} C_{1}^{1}$ be the torus with one outgoing and one incoming boundary. Since the ring structure is determined by $\tilde{Z}$ on a pair of pants, one can piece together 2 pairs of pants and obtain ${ }^{1} C_{1}^{1}$. For future references, denote $\alpha$ (the diagonal matrix) ${ }^{1} \mu_{1}^{1}=\tilde{Z}\left({ }^{1} C_{1}^{1}\right)$.

4.4. Family topological field theories. A FTFT assigns a family of topological $(m, n)$-pointed 2 - $\operatorname{dim}_{\mathbb{R}}$ surfaces $\mathcal{C} \rightarrow B$ a cohomology class $\bar{Z}(B) \in H^{*}\left(B, \operatorname{Hom}\left(A^{m}, A^{n}\right)\right)$, where $A^{m}$ are, in general, local systems on $B . \bar{Z}$ must satisfy two additional properties, in addition to being a "fiberwise TFT:"

(1) functoriality with respect to the (topological) base change;

(2) a strong gluing axiom. 
The first property ensures that all FTFTs are pullbacks of the universal setting over the classifying spaces. The second property requires a little more explanation.

The gluing requires parameterizations of boundary circles, instead of the punctures. The strong gluing axiom asserts that the gluing axiom has to hold for any lifting to families with parameterized boundaries. The $(m, n)$ pointed family can be lifted to a family of $m$ incoming and $n$ outgoing circles in the following way. Take the torus bundle $\tilde{B} \rightarrow B$ with fiber $\left(S^{1}\right)^{m} \times$ $\left(S^{1}\right)^{n}$ the product of unit tangent spaces at the marked points. (Since the space of Riemannian metrics is contractible, the choice of the metric does not matter.) Because Diff $+\left(S^{1}\right)$, the orientation-preserving diffeomorphisms, is homotopy equivalent to its subgroup of rigid rotations, $\tilde{B}$ is homotopy equivalent to the base space of the corresponding family with parameterized circle boundaries. Note that $\mathcal{C}$ allows nodal singularities.

Teleman classified all FTFTs for which the fiberwise TFT is generically semisimple.

Teleman established this classification in 3 steps.

Step 1. Classification of FTFTs $\tilde{Z}$ associated to smooth families of surfaces with parameterized boundaries.

Step 2. Going from parameterized boundaries to punctures. This requires a new piece of information, which is denoted $E(\psi)$. Roughly, at each outgoing boundary circle, $E(\psi)$ (or $E(\psi)^{-1}$ for an incoming circle) "transforms" the parameterized boundary to a puncture.

Step 3. Allowing nodal (stable) degenerations of curves. The corresponding FTFT is denoted $\bar{Z}$. $\bar{Z}$ requires again one new piece of data: $L:=\bar{Z}$ (pinched cylinder). In fact, this classification can be generalized to, what Teleman calls "Lefschetz theory", where the family of curves does not have to be stable, and with more general gluing axioms. Interested readers are referred to Teleman's original article $[\mathbf{T}]$.

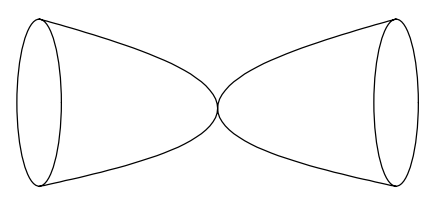

pinched cylinder

Teleman proved that $\tilde{Z}, E(\psi)$, and $L$ are sufficient to "piece together" $\bar{Z}$. Roughly, one can replace punctures by parameterized boundaries via $E(\psi)$, and smooth the nodes via $L$. The remaining theory is then $\tilde{Z}$. Among the 3 steps, Step 1 involves the aforementioned Harer stability and Madsen-Weiss Theorem and is most relevant to this article's audience. In the remaining of this section, we will give some explanation of Step 1. But we first give a short digression on how Teleman's classification proves Givental's conjecture. 
4.5. Proof of Givental's conjecture. We will use the following terminology proposed in $[\mathbf{L 4}]$.

A geometric Gromov-Witten theory (GWT) is the "usual" GWT constructed from moduli spaces of stable maps of all genera. Each geometric GWT is encoded in a single generating function

$$
\tau_{G W}:=\exp \left(\sum_{g=0}^{\infty} \hbar^{g-1} F_{g}\right)
$$

where $F_{g}$ is the genus $g$ generating function of GW invariants.

An axiomatic GWT is the one constructed from Givental's quantization formalism, when the Frobenius structure is semisimple. Roughly, the generating function of an axiomatic GWT is defined to be

$$
\tau_{G}:=\hat{\mathrm{O}} \tau^{N p t},
$$

where $\tau^{N p t}$ is the $N$ copies of Witten-Kontsevich generating function (or equivalently GWT of $N$ points), and $\hat{O}$ is an operator defined by quantizing a quadratic functions defined via genus zero GW invariants (or equivalently the Frobenius structure). In the above formula, $N$ is the dimension of the Frobenius structure or, in the geometric case, the dimension of the cohomology group.

When the Frobenius structure is semisimple, one can ask whether $\tau_{G}$ is equal to $\tau_{G W}$. The equality is termed Givental's conjecture. The conjecture in particular implies that all higher genus GW invariants can be reconstructed from genus zero invariants, as $\hat{O}$ involves only genus zero data.

One can prove that $\tau_{G}$ can be written as

$$
\tau_{G}=\exp \left(\sum_{g=0}^{\infty} \hbar^{g-1} G_{g}\right) .
$$

Furthermore, it can be shown without much difficulty that $G_{0}=F_{0}$, which implies that the genus zero truncation of Givental's conjecture holds. See $[\mathbf{L P}$, Part II $]$ for details.

Teleman's classification implies Givental's conjecture. Unfortunately, the brief account given here is mostly redundant for those who are familiar with the basic definitions. A meaningful explanation of this involves the detailed construction of Givental's quantization formalism and is beyond the scope of this article. Interested readers are referred to Teleman's original paper $[\mathbf{T}]$.

First of all, it is easy to see that axiomatic GWTs are FTFT. For those who are familiar with the definition of cohomological field theory, GWTs are CohFTs and CohFTs satisfy the axioms of FTFTs. In fact, Teleman's classification implies a stronger version of Givental's conjecture: The corresponding axiomatic and geometric CohFTs are equal. Indeed, CohFTs yield cohomology classes on $\overline{\mathcal{M}}_{g, n}$, and the integration of these classes (with monomials of $\psi$-classes) gives $\mathrm{GW}$ invariants. 
To prove Givental's conjecture, Teleman traces through Givental's quantization formalism and shows that all semisimple GWTs can be reconstructed from the genus zero data, and from known facts about the tautological classes of the moduli space of curves. (Very naively speaking, one can see all discussion in this section involves only genus zero data and moduli of curves.) Since axiomatic and geometric theories are, by construction, identical in genus zero, the proof is then complete.

4.6. Wheeled PROP structure. Let ${ }^{q} M_{g}^{p}$ be the moduli space of the Riemann surfaces of genus $g$ with $p$ incoming and $q$ outgoing marked points. Let ${ }^{q} \tilde{M}_{g}^{p}$ be the torus bundle over ${ }^{q} M_{g}^{p}$ as discussed earlier. Homotopically, ${ }^{q} M_{g}^{p} \sim B \Gamma_{g}^{p+q}$, where $\Gamma_{g}^{p+q}$ is the corresponding mapping class group, and one can think of ${ }^{q} M_{g}^{p}$ as a classifying space of bordered Riemann surfaces of genus $g$ with $p$ incoming and $q$ outgoing parameterized boundaries. Gluing the outgoing boundary circles of a surface with incoming ones of another gives the structure of a PROP on ${ }^{q} \tilde{M}_{g}^{p}$. Roughly, a PROP is something like an operad, but with multiple outputs. ${ }^{1}$ In addition to the PROP structure, it also allows contraction, via gluing boundary circles of the same surface. The additional contraction operation makes it a wheeled PROP [MMS].

4.7. Harer stability. We recall Harer stability. For the notational convenience, we will not distinguish the outgoing and incoming boundary, but use $r:=p+q$. Let $\tilde{M}_{g, r}$ be the (homotopy type of the) moduli space of oriented surfaces of genus $g$, and $r$ boundary circles. (The number of punctures will be fixed during the discussion, and will be omitted from the notations.) Let $C_{g, r}$ be a bordered oriented surface of genus $g$ with $r$ boundary circles. Define three operations

$$
\begin{array}{lrl}
\phi_{1}: C_{g, r} \rightarrow C_{g+1, r-1}, & r \geq 2, \\
\phi_{2}: C_{g, r} \rightarrow C_{g, r+1}, & r \geq 1, \\
\phi_{3}: C_{g, r} \rightarrow C_{g+1, r-2}, & r \geq 2
\end{array}
$$

as follows: $\phi_{1}$ is defined by gluing a pair of pants along 2 boundary circles; $\phi_{2}$ is defined by gluing a pair of pants along 1 boundary circle; $\phi_{3}$ is defined by gluing 2 boundary circles together.

\footnotetext{
${ }^{1}$ The formal definition of PROP, or product and permutation category, can be found in, e.g., [Ma]. Briefly, a PROP $(P, *, S, 1)$ is a symmetric strict monoidal category such that

(1) the objects $O b(P)$ are identified with the set $\mathbb{Z}_{\geq 0}$;

(2) the product satisfies $m * n=m+n$, for any $m, n \in O b(P)$ (hence the unit $1=0)$;

(3) $S$ is the permutation symmetry;

(4) each hom-set $\operatorname{Mor}_{P}(m, n)$ is a $k$-module and the operations of the monoidal category $P$ are compatible with this $k$-linear structure.
} 
Theorem 4.2 (Harer stability). $\left(\phi_{1}\right)_{*}: H_{k}\left(\tilde{M}_{g}^{r}\right) \rightarrow H_{k}\left(\tilde{M}_{g+1}^{r-1}\right)$, and similarly $\left(\phi_{2}\right)_{*}$ and $\left(\phi_{3}\right)_{*}$, are isomorphisms for $k \leq[\mathrm{g} / 3]$ (the stable range).

The stable range can be improved, but we only need this estimate.

Thanks to Harer stability, one can talk about $H_{k}\left(M_{\infty}\right)$. Here $r$ is redundant in the stable range by a combination of $\phi_{i}$ 's, and hence $\tilde{M}_{\infty}=M_{\infty}$.

4.8. Classification of ${ }^{1} \tilde{Z}$. We will first study ${ }^{1} \tilde{Z}$, i.e., FTFT with one outgoing and no incoming boundary. One can increase genus by applying the composition $\phi_{2} \circ \phi_{1}$. By semisimplicity, the effect of this operation on ${ }^{1} \tilde{Z}_{g}$ is the multiplication by (the diagonal matrix) $\alpha$. Therefore, ${ }^{1} \tilde{Z}_{g+G}=\left(\alpha^{G}\right)^{1} \tilde{Z}_{g}$ in the stable range and $\left(\alpha^{-G}\right)^{1} \tilde{Z}_{G}$ stabilizes to $\tilde{Z}^{+} \in H^{*}\left(M_{\infty}\right)$.

Proposition 4.3. (i) The degree zero component of $\tilde{Z}^{+} \in H^{*}\left(M_{\infty}\right)$ is the identity.

(ii) $H^{*}\left(M_{\infty}\right)$ carries a Hopf algebra structure.

(iii) $\log \tilde{Z}^{+}=\sum_{l \geq 1} a_{l} \kappa_{l}$, where $a_{l} \in A$.

(i) can be seen by restricting our family of oriented smooth surfaces to a point, and apply base change property: For an inclusion $\iota: p t \rightarrow M$, the pullback in cohomology in degree zero $\iota^{*}: H^{0}(M) \rightarrow H^{0}(p t)$ induces isomorphism on degree zero component of $\tilde{Z}^{+}$.

(ii) is a consequence of the wheeled PROP structure.

(iii) is a consequence of the wheeled PROP structure, gluing axiom, and Mumford's conjecture. We will explain the idea now.

An element in a Hopf algebra is called primitive if $\Delta(x)=1 \otimes x+x \otimes 1$. It is group-like if $\Delta(g)=g \otimes g$. It is easy to check that group-like elements are exponentials of primitive elements.

LEMMA 4.4. $\tilde{Z}^{+}$is a group-like element.

This follows from the monoidal structure (in PROP) on $M_{\infty}$ defined by gluing two surfaces into a pair of pants:

$$
\tilde{M}_{g_{1}}^{1} \times \tilde{M}_{g_{2}}^{1} \rightarrow \tilde{M}_{g_{1}+g_{2}}^{1} .
$$

Note that the multiplicative factor $\alpha^{-\left(g_{1}+g_{2}\right)}$ is consistent on both sides. Therefore, $\log \tilde{Z}^{+}$is a primitive element. Now, Madsen-Weiss's theorem:

Theorem 4.5 (Mumford's conjecture, [MW]).

$$
H^{*}\left(M_{\infty}, \mathbb{Q}\right)=\mathbb{Q}\left[\kappa_{0}, \kappa_{1}, \kappa_{2}, \ldots\right] .
$$

Thanks to the polynomial structure of the Hopf algebra, the only primitive elements are $\sum_{l \geq 0} a_{l} \kappa_{l}$. Applying Proposition 4.3 (i), $a_{0}=0$ and $\log \tilde{Z}^{+}=\sum_{l \geq 1} a_{l} \kappa_{l}$.

Note that the converse of the statement also holds: All elements of the form $\sum_{l \geq 1} a_{l} \kappa_{l}$ serve as FTFT on smooth surfaces with parameterized boundaries. This completely classifies $\log \tilde{Z}^{+}$and hence ${ }^{1} \tilde{Z}$. 
4.9. The final steps: Classifying ${ }^{q} \tilde{Z}^{p}$. One can increase genus by gluing ${ }^{1} C^{1}$, and can increase $p$ and $q$ by gluing a genus zero surface with $p+1$ inputs and $q$ outputs to the existing one output. The latter gives a map from ${ }^{1} \tilde{M}_{g}$ to ${ }^{q} \tilde{M}_{g}^{p}$. Thanks to Harer stability, both operations are homology equivalences in the stable range. By functoriality of base change, ${ }^{q} \tilde{Z}_{g}^{p}$ is determined by its pullback to ${ }^{1} \tilde{M}_{g}$. On ${ }^{1} \tilde{M}_{g}$, this can be seen as feeding the output of ${ }^{1} \tilde{M}_{g}$ into ${ }^{q} \mu^{p+1}: A^{\otimes p+1} \rightarrow A^{\otimes q}$. Therefore:

COROLlaRY 4.6. ${ }^{q} \tilde{Z}_{g}^{p}$ is diagonal in the (tensor power of the) normalized basis, and the entry for $\tilde{\epsilon}_{i}^{\otimes p} \mapsto \tilde{\epsilon}_{i}^{\otimes q}$ is $\Delta_{i}^{\chi / 2} \exp \left(\sum_{l \geq 1} a_{i l} \kappa_{l}\right)$.

This completes the classification of $\tilde{Z}$.

\section{Witten's conjecture on $r$-spin curves}

In this section, we briefly describe Faber-Shadrin-Zvonkine's proof [FSZ] of Witten's conjecture on $r$-spin curves. This conjecture also follows from combining Teleman's more general result in the previous section, and Givental's result in $[\mathbf{G i 3}]$. [FSZ] takes a different approach.

Semisimplicity of the Frobenius structure is tacitly assumed throughout this section.

5.1. Overview. Witten's conjecture states that a certain kind of Gromov-Witten theory can be constructed from $r$-spin curves, whose moduli spaces are certain branched covers of $\overline{\mathcal{M}}_{g, n}$. Furthermore, the generating function $\tau_{W}$ constructed from Witten's correlators satisfies the GelfandDickey hierarchy.

The proof has several ingredients. Here we explain one aspect of the proof, which is closer to our own.

Step 0. Witten [W2] and Jarvis-Kimura-Vaintrob [JKV] show, building on Jarvis' earlier work $[\mathbf{J} 1, \mathbf{J 2}]$, that the genus zero truncation of the conjecture holds. Several rigorous constructions of Witten's correlators for all genera were defined (e.g., [Mo, PV]).

Step 1. The first author proposed to prove Witten's conjecture via a version of Givental's conjecture: First, show that the axiomatic generating function $\tau_{G}$ satisfies Gelfand-Dickey hierarchy. Then, show

$$
\tau_{W}=\tau_{G}
$$

where $\tau_{W}$ is Witten's generating function.

Step 2. Givental showed that $\tau_{G}$ satisfies Gelfand-Dickey hierarchy [Gi3].

Step 3. The first author proved a $g \leq 2$ truncation of (5.1) by introducing an algorithm, termed invariance constraints, to compute universal relations in axiomatic GWT. The universal relations are, be definition, the relations which hold for all axiomatic GWTs. Furthermore, it is shown that the tautological relations are universal relations $[\mathbf{L} 1, \mathbf{L} 2, \mathbf{L 3}]$. 
Step 4. Faber-Shadrin-Zvonkine proved (5.1) in full generality by showing a reconstruction theorem which allows one to reduce the comparison of the geometric GWT and the axiomatic GWT to a genus zero version. It can be considered as a special case of Teleman's theorem in the previous section. This proof uses the idea of invariance constraints and Ionel's theorem $[\mathbf{I}]$, and is entirely different from Teleman's approach.

It is worth noting that Witten's conjecture is related to the Frobenius structure of type $A_{r}$ singularities. A generalization to type $D$ and $E$ has been carried out by H. Fan, T. Jarvis, and Y. Ruan (in preparation).

We will briefly explain Steps 0, 3 and 4.

5.2. Witten's correlators. An $r$-spin structure on a smooth curve $C$ of genus $g$ with $n$ marked points $x_{i}$ is a line bundle $L$ together with the identification

$$
L^{\otimes r}=K_{C}\left(\sum_{i} m_{i} x_{i}\right),
$$

where $0 \leq m_{i} \leq r-1$ are integers such that $r \mid\left(2 g-2-\sum_{i} m_{i}\right)$. The moduli space of $r$-spin structures has a natural compactification $\overline{\mathcal{M}}_{g, m}^{1 / r}$ with a natural morphism $\pi$ to $\overline{\mathcal{M}}_{g, n}$, which is a degree $r^{2 g-1}$ "branched cover". Note that there is a global automorphism (multiplication by $r$ th roots of unity) of any point in $\overline{\mathcal{M}}_{g, n}^{1 / r}$. The degree is $r^{2 g}$ for the corresponding coarse moduli spaces.

Witten's correlators are defined by a virtual fundamental class construction $[\mathbf{M o}, \mathbf{P V}, \mathbf{C d}]$ on $\overline{\mathcal{M}}_{g,\left(m_{1}, \ldots, m_{n}\right)}^{1 / r}$

$$
\int_{\left[\overline{\mathcal{M}}_{g,\left(m_{1}, \ldots, m_{n}\right)}^{1 / r}\right] \operatorname{vir}} \pi^{*} \Psi,
$$

where $\Psi$ is a monomial of $\psi$-classes on $\overline{\mathcal{M}}_{g, n}$. By the projection formula, it is equal to $\int_{\left[\overline{\mathcal{M}}_{g, n}\right]} c_{W} \Psi$, where $c_{W}(m) \cap \overline{\mathcal{M}}_{g, n}:=\pi_{*}\left(\left[\overline{\mathcal{M}}_{g, a}^{1 / r}\right]^{\text {vir }}\right)$. (Caution: This is slightly different from the definition of $c_{W}$ in $[\mathbf{F S Z}]$.)

5.3. Invariance constraints and universal relations. The discussion here in intended to get across general ideas, and will be imprecise. Interested readers may consult $[\mathbf{L} \mathbf{4}, \mathbf{L P}]$ or Givental's original papers ([Gi3] and references therein) for details.

The starting point of this is Givental's axiomatic (semisimple) GWTs. A major breakthrough in Givental's discovery is that the "moduli space" of Frobenius manifolds (or equivalently genus zero axiomatic theories) of a fixed dimension has an action by a "twisted loop group", or Givental's group. Furthermore, all semisimple theories form a single orbit under this group action. One of the simplest semisimple theories of dimension $N$ is the GWT of $N$ points, which is given by $N$ copies of GWT of one point. This 
implies that all semisimple axiomatic theories can be obtained by a group action on GWT of $N$ points. That is, for each semisimple axiomatic theory $T$, one can find an element $O_{T}$ in Givental's group such that $T$ is equal to $O_{T}$ acting on the moduli point defined by GWT of $N$ points. $O_{T}$ is uniquely determined if a "homogeneity" condition holds. We shall not go further into the details, but only point out that Witten's generating function $\tau_{W}$ satisfies the homogeneity condition.

A geometric GWT involves all genera and is encoded in a single generating function $\tau_{G W}$ as in (4.1). For axiomatic theories, the generating functions $\tau_{G}$ are obtained via "quantizing" $O_{T}$. $\hat{O}_{T}$ is an operator which then acts on the generating function of GWT of $N$ points $\tau^{N p t}$ as in (4.2).

One may define a universal relation to be an equation of tautological classes which holds for all axiomatic GWTs. In particular it must contain tautological relations for moduli of curves as it has to hold for $\tau^{N p t}$. To show that a tautological relation for moduli curves holds for all axiomatic GWTs, we can check whether it is "invariant" under the action of $\hat{O}_{T}$ for all axiomatic GWTs $T$. In fact, since $O_{T}$ 's form Givental group, we only have to check the invariance at the level of Lie algebra. This gives very strong constraints on the form of the possible universal relations.

It was checked that all tautological equations are universal, $[\mathbf{L 2}]$. A simple geometric proof was later discovered by Faber-Shadrin-Zvonkine and independently R. Pandharipande and the first author (see [FSZ, §3]).

5.4. The Faber-Shadrin-Zvonkine Uniqueness Theorem. Let $\mu^{i}$ be partitions of $d$. Let $H_{g}\left(\mu^{1}, \mu^{2}\right)$ be the moduli space of Hurwitz covers of degree $d$ from genus $g$ curves to $\mathbb{P}^{1}$, with fixed profiles $\left(\mu^{1}, \mu^{2}\right)$ at $(0, \infty)$ in $\mathbb{P}^{1}$, and otherwise simple ramification. Let $\bar{H}_{g}\left(\mu^{1}, \mu^{2}\right)$ be the compactification by admissible covers. There is a natural morphism

$$
\rho: \bar{H}_{g}\left(\mu^{1}, \mu^{2}\right) \rightarrow \overline{\mathcal{M}}_{g, \sum_{i}} l\left(\mu^{i}\right)
$$

by forgetting the covering maps and stabilizing the domain curves.

One can consider variations of the above constructions. First, one can allow partitions $\mu^{i}=\left(\mu_{1}^{i}, \mu_{2}^{i}, \ldots\right)$ to contain zeros, which correspond to marked unramified points. Second, $\rho$ can be composed with forgetful maps (by forgetting marked points.) For the purpose of this section, we are only interested in forgetting points which are not marked unramified points. By abusing notation, we will denote them by $\rho: \bar{H}_{g} \rightarrow \overline{\mathcal{M}}_{g, n}$. Let $p:=$ $\sum_{i} l\left(\mu^{i}\right)-n$. (Note that a marked unramified point increases the length by 1.) We will call the images of $\rho$ double Hurwitz cycles.

Witten's correlators, by definition, can be expressed as integrals of the following type

$$
\int_{\overline{\mathcal{M}}_{g, n}} c_{W} \Psi
$$


where $\Psi$ is a polynomial of $\psi$-classes. Also, by construction, (complex) degree of $\Psi$ is at least $g$. Now Ionel's theorem [I] states that any monomials in $\psi$ and $\kappa$ of Chow degrees greater than $g-1$ can be represented as a linear combination of classes of the form

$$
q_{*}\left(D H C_{1} \times D H C_{2} \times \ldots\right)
$$

where $q: \prod_{i} \overline{\mathcal{M}}_{g_{i}, n_{i}} \rightarrow \overline{\mathcal{M}}_{g, n}$ is the gluing morphism and $D H C_{i}$ is a certain double Hurwitz cycle in $\overline{\mathcal{M}}_{g_{i}, n_{i}}$. Since it is known [FP3] that double Hurwitz cycles are tautological, and tautological classes are linearly generated by monomials of $\psi$ and $\kappa$ classes on (the vertices of) the dual graphs, the above arguments imply that any monomial in $\psi$ and $\kappa$ on $\overline{\mathcal{M}}_{g, n}$ of Chow degrees at least $g$ can be represented as a linear combination of dual graphs with at least one edge. This is called g-reduction in [FSZ].

By $g$-reduction on $\Psi$ and the splitting principle of $c_{W}$, Witten's correlators can then be reduced, with simple dimension counts and manipulations of tautological classes, to genus zero correlators.

Note that the above arguments apply equally to axiomatic and geometric theories. For axiomatic theories, one has to show that all tautological relations holds, so that the same form of $g$-reduction applies. As explained in the previous subsection, this is accomplished by showing they satisfy the invariance constraints.

Now, the fact that both theories have

(1) the same reduction to genus zero correlators, and

(2) the same genus zero correlators

implies the geometric theory equals the axiomatic one. This, combined with Givental's theorem [Gi3] then proves Witten's conjecture.

\section{References}

[CLL] L. Chen, Y. Li, and K. Liu, Localization, Hurwitz numbers and the Witten conjecture, preprint 2006, math.AG/0609263.

[Cd] A. Chiodo, The Witten top Chern class via K-theory, J. Algebraic Geom. 15 (2006), no. 4, 681-707.

[CK] D. Cox and S. Katz, Mirror Symmetry and Algebraic Geometry, Mathematical surveys and Monographs 68, Amer. Math. Soc., Providence, RI, 1999.

[ELSV1] T. Ekedahl, S. Lando, M. Shapiro, and A. Vainshtein, On Hurwitz numbers and Hodge integrals, C. R. Acad. Sci. Paris Sér. I Math. 328 (1999), 1175-1180.

[ELSV2] T. Ekedahl, S. Lando, M. Shapiro, and A. Vainshtein, Hurwitz numbers and intersections on moduli spaces of curves, Invent. Math. 146 (2001), 297-327.

[F1] C. Faber, A conjectural description of the tautological ring of the moduli space of curves, in Moduli of Curves and Abelian Varieties, 109-129, Aspects Math., E33, Vieweg, Braunschweig, 1999.

[F2] C. Faber, Algorithms for computing intersection numbers on moduli spaces of curves, with an application to the class of the locus of Jacobians, in New trends in algebraic geometry (Warwick, 1996), 93-109, London Math. Soc. Lecture Note Ser., 264, Cambridge Univ. Press, Cambridge, 1999.

[FP1] C. Faber and R. Pandharipande, Logarithmic series and Hodge integrals in the tautological ring, Michigan Math. J. (Fulton volume) 48 (2000), 215-252. 
[FP2] C. Faber and R. Pandharipande, Hodge integrals, partition matrices, and the $\lambda_{g}$ conjecture, Ann. Math. (2) 157 (2003), no. 1, 97-124.

[FP3] C. Faber and R. Pandharipande, Relative maps and tautological classes, J. Eur. Math. Soc. 7 (2005), no. 1, 13-49.

[FSZ] C. Faber, S. Shadrin, and D. Zvonkine, Tautological relations and the $r$-spin Witten conjecture, arXiv:math/0612510.

[GeP] E. Getzler and R. Pandharipande, Virasoro constraints and the Chern classes of the Hodge bundle, Nuclear Phys. B 530 (1998), 701-714.

[Gi1] A. Givental, Semisimple Frobenius structures at higher genus, IMRN 2001, no. $23,1265-1286$.

[Gi2] A. Givental, Gromov-Witten invariants and quantization of quadratic Hamiltonians, Dedicated to the memory of I. G. Petrovskii on the occasion of his 100th anniversary, Mosc. Math. J. 1 (2001), no. 4, 551-568, 645.

[Gi3] A. Givental, $A_{n-1}$ singularities and $n K d V$ hierarchies, Dedicated to Vladimir I. Arnold on the occasion of his 65th birthday, Mosc. Math. J. 3 (2003), no. 2, 475-505, 743.

[GJ1] I. P. Goulden and D. M. Jackson, The number of ramified coverings of the sphere by the double torus, and a general form for higher genera, J. Combin. Theory A 88 (1999), 259-275.

[GJ2] I. P. Goulden and D. M. Jackson, The KP hierarchy, branched covers, and triangulations, Adv. Math. 219 (2008), no. 3, 932-951.

[GJV1] I. P. Goulden, D. M. Jackson, and R. Vakil, Towards the geometry of double Hurwitz numbers, Adv. Math. (Artin issue) 198 (2005), 43-92.

[GJV2] I. P. Goulden, D. M. Jackson, and R. Vakil, A short proof of the $\lambda_{g}$-conjecture without Gromov-Witten theory: Hurwitz theory and the moduli of curves, submitted for publication, preprint 2007, earlier version math.AG/0604297.

[GJV3] I. P. Goulden, D. M. Jackson, and R. Vakil, The moduli space of curves, double Hurwitz numbers, and Faber's intersection number conjecture, submitted for publication, preprint 2006, math.AG/0611659.

[GV1] T. Graber and R. Vakil, On the tautological ring of $\overline{\mathcal{M}}_{g, n}$, in Proceedings of the Seventh Gökova Geometry-Topology Conference 2000, International Press, 2000.

[GrV2] T. Graber and R. Vakil, Relative virtual localization and vanishing of tautological classes on moduli spaces of curves, Duke Math. J. 130 (2005), no. 1, 1-37.

[HLo] R. Hain and E. Looijenga, Mapping class groups and moduli spaces of curves, Proc. Sympos. Pure Math. 62 Part 2, pp. 97-142, Amer. Math. Soc., Providence, RI, 1997.

[MS] K. Hori, S. Katz, A. Klemm, R. Pandharipande, R. Thomas, C. Vafa, R. Vakil, and E. Zaslow, Mirror Symmetry, Clay Math. Inst., Amer. Math. Soc., 2002.

[I] E. Ionel, Topological recursive relations in $H^{2 g}\left(\mathcal{M}_{g, n}\right)$, Invent. Math. 148 (2002), no. 3, 627-658.

[J1] T. Jarvis, Geometry of the moduli of higher spin curves, Internat. J. Math. 11 (2000), no. 5, 637-663.

[J2] T. Jarvis, Picard Group of the Moduli of Higher Spin Curves, New York Journal of Mathematics, 7 (2001), 23-47.

[JKV] T. Jarvis, T. Kimura, and A. Vaintrob, Moduli spaces of higher spin curves and integrable hierarchies, Compositio Math. 126 (2001), no. 2, 157-212.

[Ka] M. E. Kazarian, KP hierarchy for Hodge integrals, Adv. Math. 221 (2009), no. $1,1-21$.

[KaLa] M. E. Kazarian and S. K. Lando, An algebro-geometric proof of Witten's conjecture, J. Amer. Math. Soc. 20 (2007), 1079-1089.

[KiLi] Y.-S. Kim and K. Liu, A simple proof of Witten conjecture through localization, preprint 2005, math.AG/0508384. 
[Ko] M. Kontsevich, Intersection theory on the moduli space of curves and the matrix Airy function, Comm. Math. Phys. 147 (1992), 1-23.

[L1] Y.-P. Lee, Invariance of tautological equations I: conjectures and applications, J. Eur. Math. Soc. 10 (2008), no. 2, 399-413.

[L2] Y.-P. Lee, Invariance of tautological equations II: Gromov-Witten theory, (appendix with Y. Iwao,) math.AG/0605708, to appear in J. Amer. Math. Soc.

[L3] Y.-P. Lee, Witten's conjecture and Virasoro conjecture up to genus two, in Gromov-Witten theory of spin curves and orbifolds, 31-42, Contemp. Math., 403, Amer. Math. Soc., Providence, RI, 2006.

[L4] Y.-P. Lee, Notes on axiomatic Gromov-Witten theory and applications, arXiv:0710.4349, to appear in the Proceedings of the 2005 AMS Summer Research Institute on Algebraic Geometry.

[LP] Y.-P. Lee and R. Pandharipande, Frobenius manifolds, Gromov-Witten theory, and Virasoro constraints, in preparation. Material needed for this paper are available from http://www . math. princeton. edu/\%7Erahulp/.

[LLZ] C.-C. M. Liu, K. Liu, and J. Zhou, A proof of a conjecture of Mariño-Vafa on Hodge integrals, J. Diff. Geom. 65 (2003), no. 2, 289-340.

[LX] K. Liu and H. Xu, A proof of the Faber intersection number conjecture, preprint 2008, arXiv:0803.2204.

[Lo1] E. Looijenga, Intersection theory on Deligne-Mumford compactifications after Witten and Kontsevich, Séminaire Bourbaki, Vol. 1992/93, Astérisque No. 216 (1993), Exp. No. 768, 4, 187-212.

[Lo2] E. Looijenga, On the tautological ring of $\mathcal{M}_{g}$, Invent. Math. 121 (1995), no. 2, 411-419.

[Ma] M. Markl, Operads and Props, preprint 2006, arXiv:math/0601129.

[MMS] M. Markl, S. Merkulov, and S. Shadrin, Wheeled PROPs, graph complexes and the master equations, J. Pure Appl. Algebra 213 (2009), no. 4, 496-535.

[MW] I. Madsen and M. Weiss, The stable moduli space of Riemann surfaces: Mumford's conjecture, Ann. of Math. (2) 165 (2007), no. 3, 843-941.

[Mi] M. Mirzakhani, Weil-Petersson volumes and intersection theory on the moduli space of curves, J. Amer. Math. Soc. 20 (2007), no. 1, 1-23.

[Mo] T. Mochizuki, The virtual class of the moduli stack of stable r-spin curves, Comm. Math. Phys. 264 (2006), no. 1, 1-40.

[Mu] D. Mumford, Toward an enumerative geometry of the moduli space of curves, in Arithmetic and Geometry, Vol. II, M. Artin and J. Tate ed., 271-328, Prog. Math. 36, Birk. Boston, Boston, MA, 1983.

[OP] A. Okounkov and R. Pandharipande, Gromov-Witten theory, Hurwitz numbers, and matrix models, Algebraic geometry-Seattle 2005. Part 1, 325-414, Proc. Sympos. Pure Math., 80, Part 1, Amer. Math. Soc., Providence, RI, 2009.

[Pa] R. Pandharipande, Three questions in Gromov-Witten theory, in Proceedings of the International Congress of Mathematicians, Vol. II (Beijing, 2002), 503-512, Higher Ed. Press, Beijing, 2002.

[PV] A. Polishchuk and A. Vaintrob, Algebraic construction of Witten's top Chern class, in Advances in algebraic geometry motivated by physics (Lowell, MA, 2000), 229-249, Contemp. Math., 276, Amer. Math. Soc., Providence, RI, 2001.

[S1] S. Shadrin, On the structure of Goulden-Jackson-Vakil formula, preprint 2008, http: //arxiv.org/abs/0810.0729.

[S2] S. Shadrin, BCOV theory via Givental group action on CohFT, preprint 2008, http://arxiv.org/abs/0810.0725.

[SZ] S. Shadrin and D. Zvonkine, Changes of variables in ELSV-type formulas, Michigan Math. J. 55 (2007), no. 1, 209-228.

[T] C. Teleman, The structure of 2D semi-simple field theories, preprint 2007, arXiv:0712.0160. 
[V] R. Vakil, The moduli space of curves and Gromov-Witten theory, Enumerative invariants in algebraic geometry and string theory, 143-198, Lecture Notes in Math., 1947, Springer, Berlin, 2008.

[W1] E. Witten, Two dimensional gravity and intersection theory on moduli space, Surveys in Diff. Geom. 1 (1991), 243-310.

[W2] E. Witten, Algebraic geometry associated with matrix models of two-dimensional gravity, in Topological methods in modern mathematics (Stony Brook, NY, 1991), 235-269, Publish or Perish, Houston, TX (1993).

Department of Mathematics, University of Utah, Salt Lake City, UT 84112-0090

E-mail address: yplee@math.utah.edu

Department of Mathematics, Stanford University, Stanford, CA 943052125

E-mail address: vakil@math.stanford.edu 
